\title{
Channel Coding Based on Power Line Communication
}

\author{
Xiaojun Chen* and Yunxiao Zu \\ School of Electronic Engineering, Beijing University of Posts and Telecommunications, Beijing, 100876, China \\ ${ }^{*}$ Corresponding Author: Xiaojun Chen. Email: 13811833130@163.com \\ Received: 17 November 2021; Accepted: 12 December 2021
}

\begin{abstract}
This paper focuses on the forward error correction (FEC), the basic parameters determination of the RS convolution code, Turbo code and the LDPC code, and the corresponding encoding and decoding algorithm in power line communication (PLC) standard. Simulation experiment which is designed for narrow-band power line communication system based on OFDM is done. The coding using RS convolution code, Turbo code and LDPC code are compared, and further it is determined that which encoding method is more suitable for power line communication in China.
\end{abstract}

Keywords: OFDM communication; RS convolution code; Turbo code; LDPC code; channel coding

\section{Introduction}

Power line distribution network is very common, and the PLC (Power Line Communication) is featured with low-cost and quick. However, the power line is disturbed by the synchronization pulse period noise, burst noise, background noise, and frequency-selective Rayleigh fading, which limits the development and application of PLC. In recent years, OFDM (Orthogonal Frequency Division Multiplexing) technique and FEC (Forward Error Collection) accelerate the development and application of PLC. These standards define PHY (Physical Layer Device), MAC (Media Access Control), and LLC (Logical Link Control) [1]. Researching to the PHY, especially to the FEC part of the PHY, has important significance to improve noise immunity and stability of the PLC networks.

\section{Power Line Communication Systems Based on OFDM}

Fig. 1 shows that the frame structure of physical layer data unit is cascaded with three parts, namely the preamble sequence (Preamble), the frame control header (FCH) and the valid data (Data). In which the effective MAC layer data needs the data transmitted by the PHY. FCH, which carries a variety of information of this transmission data, such as mapping, the sub- carrier selection, symbols, etc., are the signalling overhead. The valid data is done IFFT (Inverse Fast Fourier Transformation) after crambling, cascade correction coding, interleaving, mapping, power and bit distribution by the DSM (Dynamic Spectrum Management) [2]. In order to decrease the multipath delay and crosstalk between sub-carriers, the cyclic prefix is added. At the same time, in order to reduce the out-off band radiation, Raised Cosine is used. Thus an OFDM signal is generated and loaded to power lines through an analog front-end (AFE). 


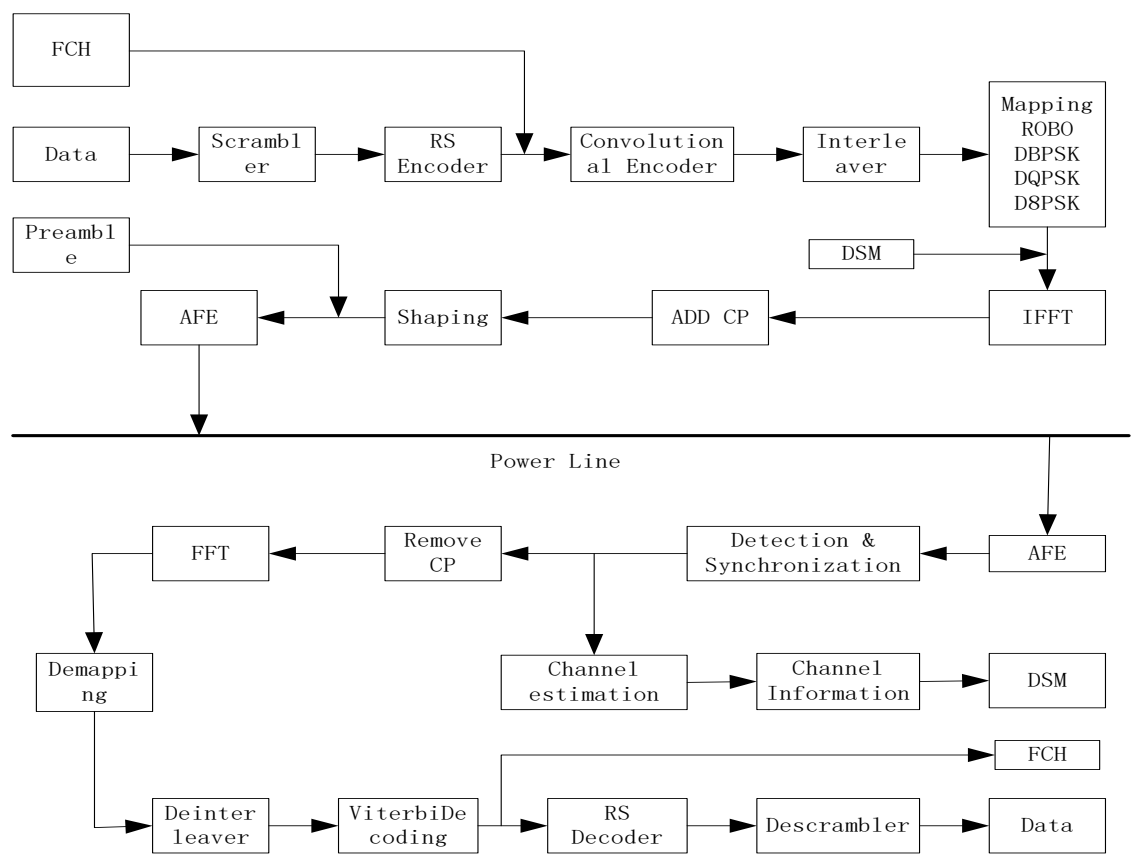

Figure 1: The diagram of NB-PLC system

By the channel estimation, the receiver detects the synchronization symbol and data, obtains the channel parameters. At the same time, by demodulating the $\mathrm{FCH}$, the way of the sub-carrier mapping, the sub-carriers selection and symbol are obtained, and then the valid data is demodulated, including demapping, deinterleaving, decoding, descrambling codes and so on [3].

\section{RS convolution Code, Turbo Code, LDPC Code Parameters}

\subsection{RS Convolution Code Parameters}

The system takes G3 PLC coding standard, using RS codes cascade with convolution codes, that is RS does outer code and convolution code does inner-code. The normal mode uses the $\operatorname{RS}(N=255, K=$ $239, T=8)$ and the Robust mode uses $\operatorname{RS}(N=255, K=247, T=4)$, the encoding rate is $1 / 2$ [4].

The code generator polynomial is:

$$
g(x)=\prod_{i=1}^{16}\left(x-\alpha^{i}\right)
$$

The field generator polynomial is:

$p(x)=x^{8}+x^{4}+x^{3}+x^{2}+1$

The most commonly used convolution code is $(2,1,7)$ encoding [5].

$$
\begin{aligned}
& A(D)=1+D^{2}+D^{3}+D^{5}+D^{6} \\
& B(D)=1+D+D^{2}+D^{3}+D^{6}
\end{aligned}
$$

\subsection{Turbo Code Parameters}

The component encoders are consisting of two $(2,1,3)$ RSC codes which have the same rate of $2 / 3$, whose transfer function $G(x)$ is:

$G(x)=\left[1, \frac{1+x^{2}}{1+x+x^{2}+x^{3}}\right]$ 
The check matrix is:

$$
H=\left[\begin{array}{lll}
0 & 1 & 1 \\
1 & 0 & 0 \\
0 & 1 & 0
\end{array}\right]
$$

The turbo interleaves parameters are shown in Table 1.

Table 1: The interleaves parameters

\begin{tabular}{llll}
\hline PB size (Octets) & $N$ & $M$ & Interleaves length $L$ \\
\hline 136 & 34 & 16 & 544 \\
\hline
\end{tabular}

The interleaving mapping function $I(x)$ is:

$I(x)=[S(x \bmod N)-(x \operatorname{div} N) \times N+L] \bmod L$, for $x=0,1, \cdots,(L-1)$

If the output information bit is even, the result of the corresponding interleaved information bits is bits 0 and 1 exchanged in pairs. So the interleaving mapping function $I(x)$ can be expressed as:

$$
\begin{gathered}
f x \bmod 2=0, \operatorname{IntData}(2 * x)=\operatorname{Data}(2 * I(x)+1) \\
\operatorname{IntData}(2 * x+1)=\operatorname{Data}(2 * I(x)) \\
f x \bmod 2=1, \operatorname{IntData}(2 * x)=\operatorname{Data}(2 * I(x)) \\
\operatorname{IntData}(2 * x+1)=\operatorname{Data}(2 * I(x)+1)
\end{gathered}
$$

\subsection{LDPC Parameters}

The LDPC coding and the structure of LDPC encoder are shown in Fig. 2 and Fig. 3, respectively.

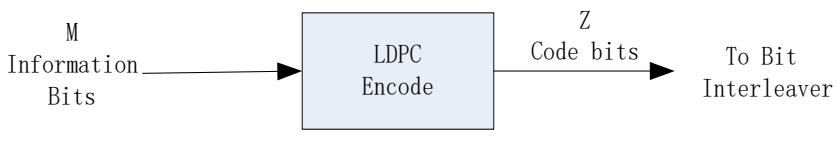

Figure 2: LDPC coding

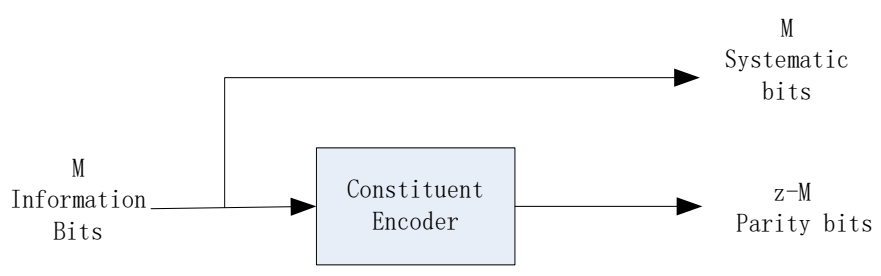

Figure 3: Structure of LDPC decoder

Fig. 2 and Fig. 3 show that: 1) The information bits(M bits) are used as the system bits(M bits), and also used as the input of the encoder in the encoding process. 2) The input of the component encoder is information bits(M bits), while the output is check bit(z-Mbits).

The coding system is defined by a limited check matrix. The check matrix is periodic and the check polynomial varies $k$. The check equations are shown as follows [1]:

When $k=0$, the check equation is:

$$
\begin{aligned}
& A_{X 1,0}(D) X_{1}(D)+B_{0}(D) P(D) \\
& =\left(D^{214}+D^{185}+1\right) X_{1}(D)+\left(D^{215}+D^{145}+1\right) P(D)=0
\end{aligned}
$$

When $k=1$, the check equation is: 


$$
\begin{aligned}
& A_{X 1,1}(D) X_{1}(D)+B_{1}(D) P(D) \\
& =\left(D^{160}+D^{62}+1\right) X_{1}(D)+\left(D^{206}+D^{127}+1\right) P(D)=0
\end{aligned}
$$

When $k=2$, the check equation is:

$$
\begin{aligned}
& A_{X 1,2}(D) X_{1}(D)+B_{2}(D) P(D) \\
& =\left(D^{196}+D^{143}+1\right) X_{1}(D)+\left(D^{211}+D^{119}+1\right) P(D)=0
\end{aligned}
$$

In the Eqs. (8)- $(10), X_{k}(D)(k=1,2, \ldots, N-1)$ is the system bit, and $P(D)$ is the check bit, $D$ is the delay operator.

The terminal of coded bits is called Tail-bits, expressed by $m_{z}$, and used for coding finished. The length of Tail-bits is determined by the information code length and the coding rate. In this article $m_{t}=440$ bits (not the check bit), and are set to 0 . Then $m_{z}$ (including the parity bits) may be obtained from the Eq. (12).

$$
\begin{array}{ll}
q=0: & m_{z}=(N-1) m_{t} \\
q \neq 0: & m_{z}=(N-1) m_{t}+(N-1) q
\end{array}
$$

where $R=(N-1) / N$, so the value of $N$ is 2 in the LDPC code, i.e., the code rate is $1 / 2$.

\section{Simulation and Results}

\subsection{Channel}

Taking the laboratory power line channel as actual channel, the solid lines in Fig. 4 and Fig. 5 are the measured frequency characteristics of the power line channel.

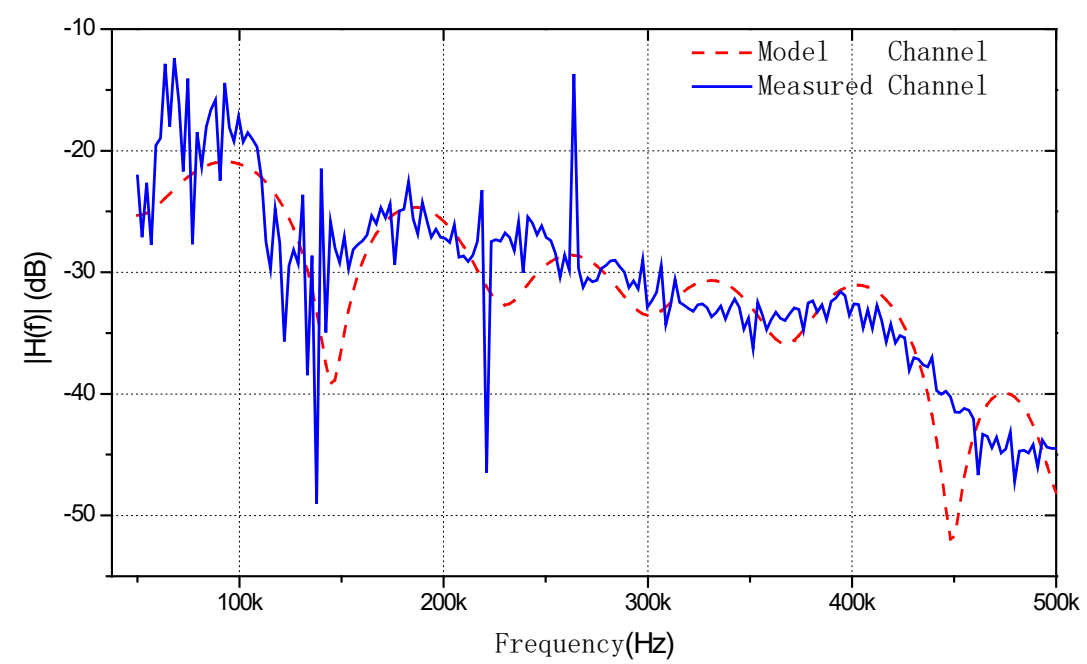

Figure 4: The measured amplitude-frequency characteristic of the power line channel

The channel data is taken from the measured amplitude-frequency characteristic in laboratory. The time domain model of the power line channel with 14 paths can be built from the channel data by Linear Minimum Mean Squared Error (LMMSE) estimation fitting, and it is shown in Fig. 6. The dashed line in Fig. 4 and Fig. 5 are the frequency characteristic of the model [6,7]. 


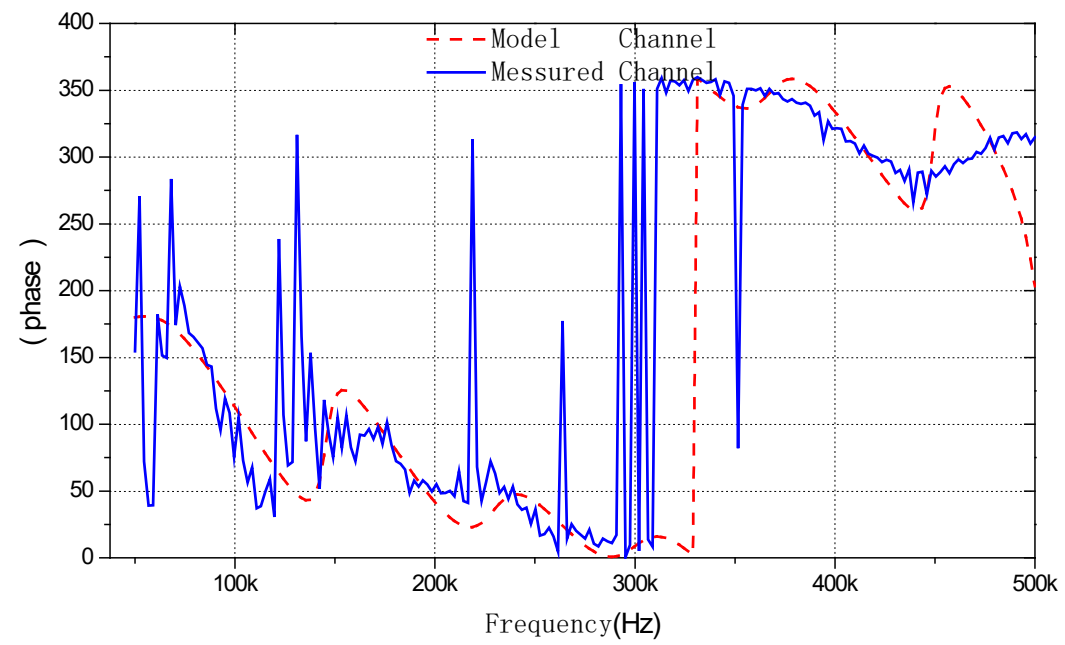

Figure 5: The measured phase-frequency characteristic of the power line channel

It can be seen from Fig. 4 and Fig. 5 that the channel of the system can simulate the amplitudefrequency parameters, phase-frequency parameters and multipath characteristics of the noise in the actual power line channel. So the multipath parameters shown in Fig. 6 are used in simulation after normalization.

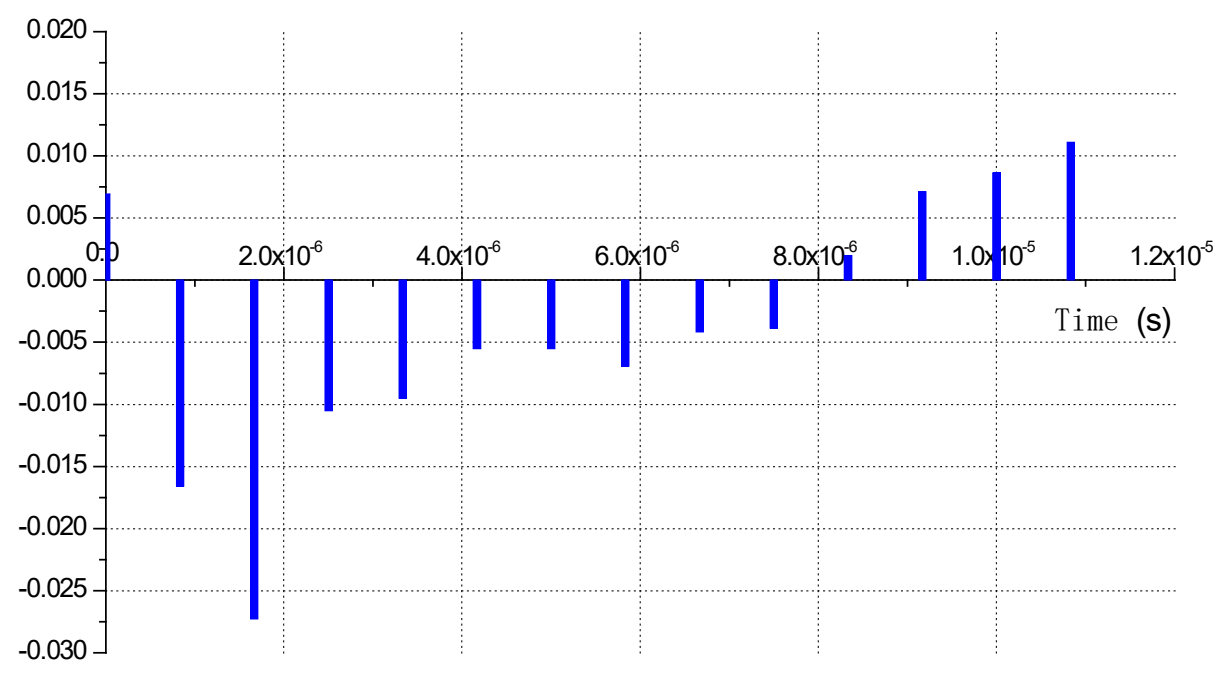

Figure 6: The power line multipath channel model

\subsection{Channel Coding}

The first channel coding is RS convolution concatenated code. Taking $\mathrm{RS}(255,239)$ code as an outer code, convolution code $(2,1,7)$ as inner code, the tail bit is 6 bits, the rate is $1 / 2$, and the decoding method of convolution code is Viterbi decoding. The second channel coding is Turbo code, the component code encoder has three registers, the selected Turbo codes have 9 tail bits, the rate is $1 / 2$, the code length $N$ is 487, and Log-MAP decoding algorithm is used with 6 iterations. The third channel encoding is LDPC code, the tail bits are 440, the rate is $1 / 2$, the code length $N$ is 918 , and Min-Sum decoding algorithm is used. Considering the complexity of the hardware implementation, the selected Turbo iteration is 4 , the Log-MAP algorithm iteration is 6 and the LDPC iteration is 12 [8-12]. 


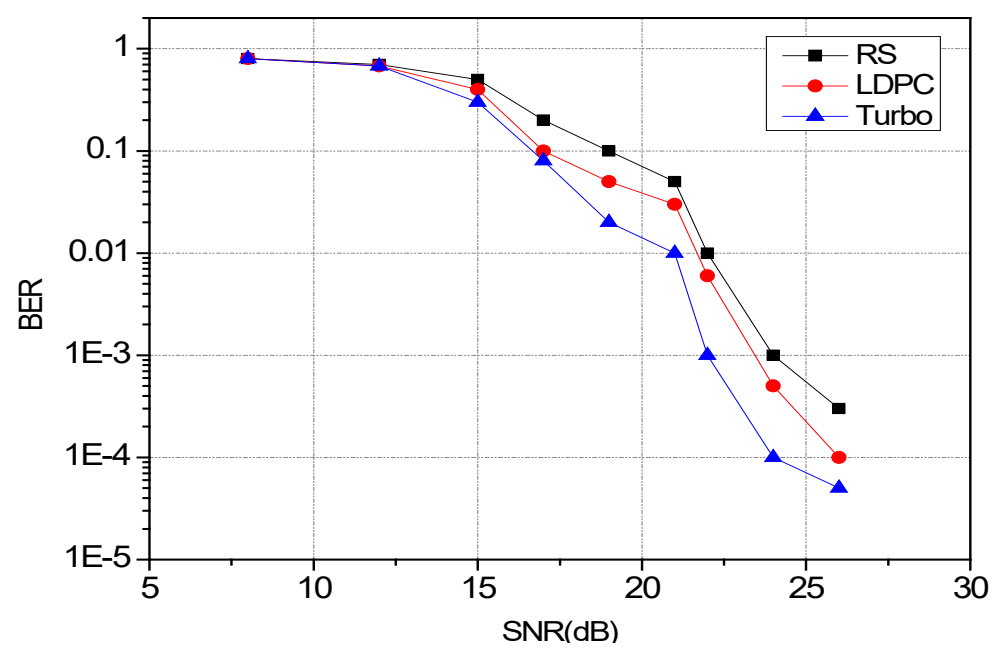

Figure 7: BER vs. SNR for three coding

In the narrowband power line communication system based on OFDM, simulations are done by using above the three coding methods respectively, the SNR vs bit error rate is shown in Fig. 7.

It can be seen from Fig. 7 that Turbo coding is better than RS convolution coding and LDPC coding. So Turbo coding is used for the narrowband power line communication system based on OFDM.

\section{Conclusions}

The parameters of RS convolution code, Turbo code and LDPC code are defined in this paper. The coding performance of the three codes is compared when they used in the narrowband power line communications system based on OFDM. Then the conclusion is achieved that respect to a given parameter RS convolution coding, LDPC coding and Turbo coding, which encoding method is more suitable for the power line communication channel in China.

Acknowledgement: This work was supported by the State Key Lab of Power System, Tsinghua University, Beijing, China (SKLD11KM05).

Funding Statement: The authors received no specific funding for this study.

Conflicts of Interest: The authors declare that they have no conflicts of interest to report regarding the present study.

\section{References}

[1] M. Hoch, "Comparison of PLC G3 and PRIME," in 2011 IEEE Int. Sym. on Power Line Communications and Its Applications, pp. 165-169, 2011.

[2] "PLC G3 Physical Layer Specification" [Online]. Available:

http://www.erdfdistribution.fr/medias/Linky/PLC_G3_Physical Layer Specification.PDF, 2011.

[3] "Draft Standard for Powerline Intelligent Metering Evolution (PRIME), PRIME Alliance Technical Working Group" [Online]. Available: http://www.prime-alliance.org/portals/0/specs/PRIMESpecv1 3 E_201005.pdf, 2010.

[4] "IEEE standard for broadband over power line networks: Medium access control and physical layer specifications" in IEEE Std 1901-2010, pp. 1-1586, 2010.

[5] K. Razazian, M. Umari and A. Kamalizad, "Error correction mechanism in the new G3-PLC specification for powerline communication," in 2010 IEEE Int. Sym. on Power Line Communications and Its Applications, pp. $50-55,2010$. 
[6] J. M. Domingo, S. Alexandres and C. Rodriguez-Morcillo, "PRIME performance in power line communication channel," in 2011 IEEE Int. Sym. on Power Line Communications and Its Applications, pp. 159-164, 2011.

[7] HomePlug Powerline Alliance, Inc. Home Plug Green PHYтм 1.1, "The Standard for Smart Grid Powerline Communications," 2012.

[8] T. Wada, "A study on the performance of turbo coding for noise environments in power lines," in IEEE International Conf. on Communications, vol. 5, pp. 3071-3075, 2003.

[9] K. Sripimanwat, "Turbo code applications: A journey from a paper to realization," National Electronics and Computer Technology Center (NECTEC), Pathumthani, Thailand, pp. 1-13, 2003.

[10] T. C. Chuah, "Adaptive robust turbo equalization for powerline communications," IEEE Transactions on Power Delivery, vol. 22, no. 4, pp. 2172-2179, 2007.

[11] N. T. Thao and D. Rzepka, "Time encoding of bandlimited signals: Reconstruction by pseudo-inversion and timevarying multiplierless FIR filtering," in IEEE Transactions on Signal Processing, vol. 69, pp. 341-356, 2021.

[12] K. Adam, A. Scholefield and M. Vetterli, "Multi-channel time encoding for improved reconstruction of bandlimited signals," in 2019 IEEE Int. Conf. on Acoustics, Speech and Signal Processing, pp. 7963-7967, 2019. 